\title{
Leonhard Eulers Wege zur Zahlentheorie
}

\author{
Maarten Bullynck
}

Mit fast hundert Aufsätzen ist der Output Leonhard Eulers zur Zahlentheorie so umfangreich, dass er nahezu unübersichtlich wird. Vom ersten Text aus dem Jahr 1732 bis die fertiggeschriebenen Aufsätze im Nachlas beim Tode Eulers in 1783, es sind mehr als 50 Jahre Arbeit am Weg zur Zahlentheorie. Ein Weg, den sich Euler eigenhändig hat anbahnen müssen. Der französische Mathematiker André Weil (1906-1998) hat Eulers zahlentheoretisches Oeuvre öfters so beschrieben': Euler sei den Weg von Pierre de Fermat aufs neue gegangen, und habe alle nicht-veröffentlichten Beweise aber auch Vermutungen Fermats selbst untersuchen, sich aneignen und beweisen müssen. ${ }^{2}$ In diesem Sinne hat erst Euler das Wenige, was Fermat überliefert hat, interpoliert, ergänzt, erweitert und für die Generation nach ihm lesbar gemacht. Aber vor allem, Euler hat die Fundamente, auf die Lagrange, Legendre und Gauss später die Zahlentheorie begründen werden, klargestellt und die wesentlichen Probleme und auch manche Lösungen formuliert.

Wenn man sich Leonhard Eulers massive Produktion anschaut, hat es allen Anschein, dass Euler fast ohne Unterbrechungen über verschiedene Fragen und Probleme der Zahlentheorie geforscht und geschrieben hat. Wie in Figur $1 \mathrm{zu}$ sehen, laufen Eulers Publikationen ununterbrochen von 1738 bis zu seinem Tod in 1783. Diese Graphik ist jedoch auf die Jahreszahlen der Veröffentlichung basiert. Macht man eine ähnliche Graphik für die Jahreszahlen der Konzeption der Ideen, welche in den Aufsätzen erst später fixiert worden sind, d.h., die Jahreszahlen, in denen Euler über ein gewisses Thema an der Akademie in Sankt-Petersburg bzw. Berlin vorgetragen hat, so sieht das Bild ganz anders aus. ${ }^{3}$

Auf einmal gibt es Struktur, Akzentuierung und Rhythmus im Schaffen Eulers. 1730 fängt die Beschäftigung an, und um 1750 und in den späten 1770er liegen die Schwerpunkte zahlentheoretischen Denkens. Die Notizbücher Eulers bestätigen diese Struktur: Die Jahre 1736 bis 1744 und die Jahre 1767 bis 1783 verzeichnen die meisten Einträge zur Zahlentheorie. ${ }^{4}$ Jede dieser Schaffensphasen lässt mit intensivem Briefwechsel, intensiver Lektüre und mit Auseinandersetzungen verknüpfen. Es wird Ziel dieses Aufsatzes zu Eulers 300. Geburtstag sein, diese Artikulation in Eulers Werk zu Zahlentheorie narrativ darzulegen. Anhand einiger ausgewählten Aufsätze werden wir die Schreibweise, Methodik und Ergebnisse Eulers skizzieren, sowie zeigen, wie Euler im wissenschaftlichen Kommunikationsnetzwerk seiner Zeit funktioniert.

1 A. Weil (1983), „L'oeuvre arithmétique d'Euler“, in: Leonhard Euler 1707-1783: Beiträge zu Leben und Werk (Basel: Birkhäuser), S. 111-134. Ebenfalls, A. Weil (1984), Number Theory: An approach through history from Hammurapi to Legendre (Basel:Birkhäuser), S. 159-296.

2 Neben Weil, siehe auch J. E. Hofmann (1961), „Über zahlentheoretische Methoden Fermats und Eulers, ihre Zusammenhänge und ihre Bedeutung", Archive for the history of exact sciences 1, S. 122-159.

3 Die Graphiken wurden mithilfe des elektronischen Euler Archive (http://www.math.dartmouth.edu/ euler/) erstellt.

4 Galina P. Matvievskaja und Helena P. Ozhigova (1983), „Eulers Manuskripte zur Zahlentheorie“, in: Leonhard Euler (1707-1783): Beiträge zu Leben und Werk. Gedenkbands des Kantons Basel-Stadtî (Basel: Birkhäuser), S. 151-160, hier 154. 

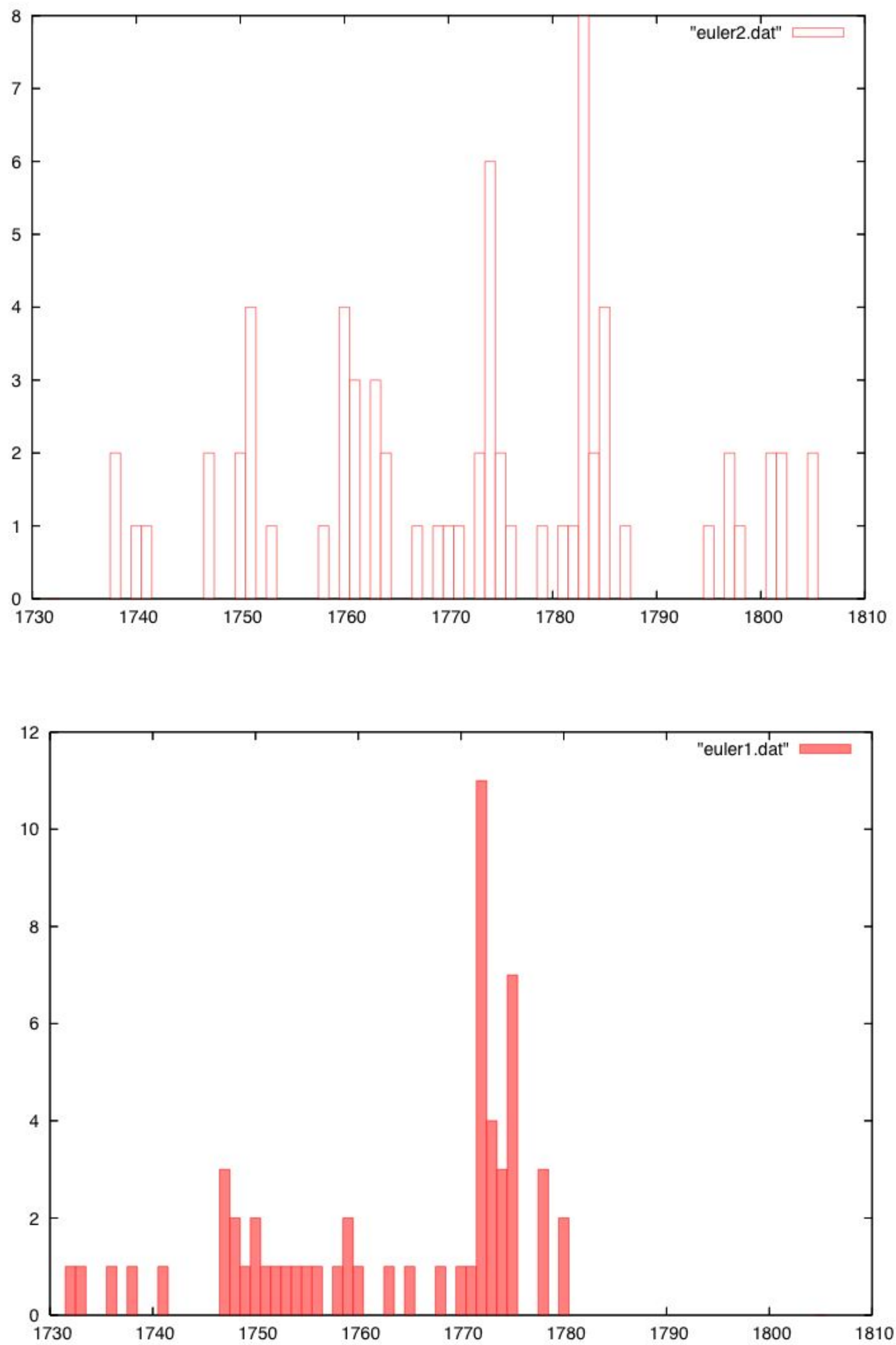


\section{Anfänge und Erinnerungen (1729-1734)}

Nach allgemeinem Konsens fängt Eulers Engagement für die Zahlentheorie Ende 1729 an, auf Anregung von seinem Briefpartner Christian Goldbach. ${ }^{5}$ Dezember 1729 suggeriert Goldbach Euler folgendes Problem: Ob Euler die Beobachtung Fermats kenne, dass all Zahlen der Form $2^{2^{n-1}}+1(n=1,2,3, \ldots)$ Primzahlen seien, aber niemand einen Beweis dieser Vermutung habe geben können. ${ }^{6}$ In einer ersten Reaktion (Januar 1730) muss Euler gestehen, er habe die Frage nicht lösen können, und er zweifle sogar, ob es legitim sei, den Satz durch blosse Induktion gelten zu lassen, da Euler selbst schon bei $2^{25}+1$ auf die Grenze seiner Rechenkraft gestoßen war. ${ }^{7}$

Das Wesentliche in der Zahlentheorie ist öfters aber, die Grenzen der Rechenkraft zu erweitern und shortcuts zu suchen. Eben in diesem Geist antwortet Goldbach (Mai 1730): Euler müsse ja diese Zahlen nicht Ziffer pro Ziffer errechnen, sondern es reiche schon mit den Resten nach Teilung zu rechnen. So weiß man, dass wenn eine Zahl N als Rest 3 hat, die Zahl 5 mal N als Rest 5 mal 3 hat. Dies ermöglicht eine große Verkürzung des Rechnens. Ein ähnlicher Trick, so bemerkt Goldbach, liege versteckt in dem von Fermat behaupteten Satz, dessen Beweis Fermat allerdings nie veröffentlich hat, dass Zahlen der Form $2^{k}+1$ immer eine Zahl der Form $2^{k i}+1$ teilen (wenn $k i$ nur keine Potenz von 2 ist). ${ }^{8}$ Euler greift sofort auf dieses neue Problem zu und erörtert in seinem nächsten Brief (Juni 1730), dass ein Beweis einer Verallgemeinerung dieses Satzes ein wichtiger Schritt zur Primzahlerkennung sein würde. Die Verallgemeinerung steht als Eulers Satz oder auch als Variante von Fermats kleinem Satz bekannt ${ }^{9}$ und lautet folgendermaßen: $a+b$ teilt immer $a^{n}+b^{n}$ (mit $n$ ungerade). Die Studien zu diesem Satz, zu dessen Beweis und zu dessen Anwendung zur Primzahlerkennung von $2^{25}+1$ bilden zusammen einen wesentlichen Teil der ersten Phase in Eulers Beschäftigung mit Zahlentheorie. ${ }^{10}$

Goldbachs Anregungen zur Zahlenthorie fielen auf fruchtbaren Boden, auch wenn Euler zu der Zeit weder Fermat noch C.G. Bachet de Méziriacs Diophant-Edition (1621)

5 Siehe z.B. die Einführung zu Leonhard Euler (1915), Opera omnia, ser. I, vol. 2, Commentationes arithmeticae, ed. Ferdinand Rudio. Leipzig: Teubner. (Weiterhin zitieren wir die Opera Omnia Serie und Band folgenderweise: OO.I.2) Ronald Calinger (in dem Aufsatz „Leonhard Euler: The First St. Petersburg Years (1727-1741)“, Historia Mathematica, Band 23 (1996), S. 121-166) relativiert dieses Bild und weist auf die Lektüren Eulers bei Johann I Bernoulli, die vor der Goldbach-Korrespondenz liegen, hin. Unser Ansatz ist dem Calingers verwandt doch geht einen anderen Weg.

6 Die Zahlen der Form $2^{2^{n}}+1$ heißen heutzetage Fermatzahlen. Sie sind mit den Zahlen der Form $2^{n}-1$, die Mersennezahlen, der Form nach verwandt. Wenn eine Mersennezahl auch eine Primzahl ist, dann ist $2^{n-1}\left(2^{n}-1\right)$ eine perfekte Zahl. Letzteres Ergebnis findet man schon in Euklids Elemente und ist ein wichtiges Stück des pythagoreischen Erbes bei Euklid. Perfekte Zahlen waren für die Pythagoreer perfekt, weil die Zahl gleich die Summe ihrer Teiler ist, z.B. 6 ist eine perfekte Zahl weil gleich $1+2+3$. Eben die Suche nach perfekten Zahlen im 16. und 17. Jh. und die Rolle der Mersennezahlen darin, haben Fermat zur Untersuchung der Zahlen $2^{2^{n}}+1$ gebracht, und auch zu seiner (falschen) Vermutung.

7 Brief II der Euler-Goldbach-Korrespondenz, in: Paul H. Fuss (ed. 1843), Correspondance mathématique et physique de quelques célèbres géomètres du XVIIlème siècle, 2 Bde. (London: Johnson Reprint Co., 1968), Band I, S.10 \& 18.

8 Fuss, Correspondance, S. 20.

9 Zur Unterscheidung von Fermats letztem Satz.

10 Der andere Teil machen die Untersuchungen Eulers zu Teilern von Zahlen der Form $a a+N b b$ aus, zu diesen quadratischen Diophantischen Problemen, oder kurz: zu quadratischen Formen, siehe weiter im Text. 
gelesen hatte. Auf andere Wege aber waren Eulers Interesse und Können schon geschärft. Wie Euler in einer Selbstbiographie berichtet, hatte sein Vater ihn schon früh „mathematisiert":

vi ich bey Zeiten von meinem Vater den ersten Untericht erhielt; und weil derselbe einer von den Discipeln des weltberühmten Jacobi Bernoulli gewesen, so tractete er mir sogleich die erste Gründe der Mathematic beizubringen, und bediente sich zu diesem End des Christophs Rudolphs Coss mit Michaels Stiefels Anmerckungen, worinnen ich mich einige Jahr mit allem Fleisch übte. ${ }^{11}$

Eben auf diese Quelle greift Euler zurück, als er einen seiner ersten veröffentlichten Aufsätze zur Zahlentheorie schreibt.

1734 präsentiert Euler der Petersburger Akademie einen Aufsatz zu Problemen mit Resten nach Teilung, gerade jener shortcut der in der elementaren Zahlentheorie von kapitaler Wichtigkeit ist. ${ }^{12} \mathrm{Zu}$ Anfang schlägt Euler vor, für die lose Kategorie der Restprobleme einen ähnlichen theoretischen und mit Beweisen unterstützten Rahmen zu schaffen wie es De la Hire und Sauveur jüngstens (1702-1705, aber 173-32 erschienen) für die magischen Quadrate getan hatten. Denn, „man findet in den gemeinen Büchern zur Arithmetik jede Menge von Problemen, zu deren vollständiger Auflösung nur mehr Fleiß und Geschicklichkeit vonnöten sind“" (S. 46). Die Klasse von Problemen, die Euler hier einheitlich behandeln und lösen will, sind Probleme der Art: Gegeben eine Anzahl $X$ von Dingen, bei Teilung durch $a, b, c, d$, usw. hat man je $A, B, C, D$, usw. Dinge übrig, was ist die Zahl $X$ ?

Nachdem Euler einige elementare Sätze bewiesen und die Lösungsmethode angegeben hat $^{13}$, zeigt er seine Methode an verschiedenen Beispielen aus der Literatur. Erstes Beispiel kommt, kein Zufall, aus Stifels Edition von Rudolffs Coss. Das Problem ist ein Zahlenrätsel:

So nu einer [...] heimlich hette im selbs verzeichnet dies zal 666, und wolt ich solte sie errathen / so sprech ich, Lieber, dividir mir dein zal durch 35 und sag mir was ubrig bleib. So müste er sagen / das nur 1 überbleibe das multiplicirte ich mit 36 (als durch meynen quotient) so bleiben die 36. die behielte ich. Zum andern hiesse ich zu sein zal auff ein newes dividiren durch 36 [...] so würde er mir müssen sagen / das 18 weren uberbliben. Deumb würde ich 1225 mit 18 multipliciren so kommen 22050. Dazu müste ich addiren das vorbehalten/ Nemlich 36. facit 22086. Das müste ich denn dividiren durch 1260. So würden 666 uberbleiben/Das ist die verburgen zal die ich solt errhaten. ${ }^{14}$

Diese Zahlenrätsel haben eine Geschichte, die gleich alt ist wie die Geschichte und Überlieferung des indisch-arabischen Zahlensystems - Rätsel und Zeichen wurden gleichzeitig in Europa (nach einer Reise aus dem alten China über Indien und schließlich über die Araber) eingeführt. Deren erste Erwähnung ist in Leonardo Pisanos (besser bekannt als Fibonacci) Liber Abaci (1202).

11 E. Fellman (1995): Leonhard Euler. Reinbeck: Rowohlt, S. 11.

12 L. Euler (1734/1740), ,Solutio problematis arithmetici de inveniendo numero, qui per datos numeros divisus relinquat data residua“, Comm. Ac. Petrop. 7, S. 46-66, auch in OO.I.2, S.18-32.

13 Nach moderner Auffassung löst Euler hier den linearen Fall der Diophantischen Gleichungen mit zwei unbekannten. D.h. löse $a x+b y=c$ mit gegebenen $a, b, c$ nach $x$ und $y$ auf, mit der Beschränkung dass $x$ und $y$ ganze Zahlen sein sollen. Vor ihm hatte Bachet de Méziriac das Problem schon vollständig gelost, siehe dazu M. Bullynck (2008), „Modular Arithmetic before C.F. Gauss. Systematisations and discussions on remainder problems in 18th century Germany“, Historia Mathematica, erscheint 2008.

14 Stifel, M. (1553), Die Coss Christoffe Rudolffs mit schönen Exempeln der Coss., Alexandrus Lutomyslensis, Königsberg, S. 15-16. 
Für den hier angeführten Fall gibt es eine besondere Regel, die Stifel selbst erfunden hat. Sie ist implizite im Originaltext, in moderner Version läuft sie folgendermaßen: Eine unbekannte Zahl lässt nach Teilung durch $n$ und $n+1$ die Reste $a$ und $b$, dann ist die unbekannte Zahl gleich dem Rest von $a(n+1)+b n^{2}$ nach Teilung durch $a b$. Euler zeigt, wie seine allgemeine Lösung als Sonderfall auch Stifels Regel enthält, mehr noch, Euler zeigt, wie man in ähnlicher Art und Weise unendlich viele solcher Spezialregeln generieren kann. Sein Aufsatz schließt mit weiteren Beispielen aus der alten Literatur von Arithmetik und Unterhaltungsmathematik: Verteilungsrätsel, Kalenderprobleme, ... Dank Eulers methodischem Ansatz lassen sich all diese Fälle mit deren Speziallösungen und -Formeln mit derselben Methode vollständig lösen, und wird die Auflistung besonderer Regeln überflüssig, weil die allgemeine Regel jetzt bekannt ist.

Simultan zur Korrespondenz mit Christian Goldbach, der Eulers Beschäftigung mit Zahlenproblemen dauernd mit neuen Fragen und Vermutungen ankurbelt, eignet Euler sich allmählich die Fachliteratur an. 1729 beginnt er, Fermats Varia opera mathematica $\mathrm{zu}$ lesen, er liest die 3 Bände der Opera vom englischen Mathematiker John Wallis (1616-1703) durch, mit einbegriffen die Korrespondenz, die Fermat mit Wallis und anderen englischen Mathematikern geführt hatte. Hauppthema dieser Korrespondenz war die Lösung der quadratischen Diophantischen Gleichung, nämlich, löse $a x^{2}+b y^{2}=c$ mit gegebenen $a, b, c$ und finde ganzzahlige $x$ und $y$, welche der Gleichung genüngen. ${ }^{15}$ Und im Prozess dieses Aneignens kommt in der Korrespondenz folgende schöne Stelle vor:

Ich bin gerade, während ich die Opera Fermats las, auf noch einen weiteren nicht uneleganten Satz gestoßen, daß alle Zahlen als die Summe von 4 Quadraten geschrieben werden können. ${ }^{16}$

Ein fast rhetorisch zu geschickt gebrachter Fund, so plötzlich beim Lesen, aber tatsächlich ein Problem, mit dem Euler sich bis zu seinem Tode beschäftigen wird, dessen Beweis zu finden, er die Ehre jedoch dem französischen Mathematiker J.-L. Lagrange lassen musste. ${ }^{17}$

In den Vierziger Jahren des 18. Jhs. beschäftigt Euler sich intensiv mit den Problemen, die er bei Fermat gefunden hat und zu denen Teillösungen bei Wallis zu finden sind. Nach Chronologie sind die Probleme vor allem: Die Primalität von $2^{2^{5}}+1$, ein Beweis für Fermats kleinen Satz, Untersuchunhen zu quadratischen Formen und ein Beweis für den 4-Quadraten-Satz. Zur Beantwortung dieser Fragen errechnet, sammelt Euler sich „Beobachtungen“, und gegen Mitte der Vierziger Jahre, gelingen ihm die Beweise der zwei ersten Probleme. Die Abhängigkeit dieser Untersuchungen untereinander und die Reihenfolge der Berechnungen und Resultate sind typisch für Eulers Arbeitsweise.

Eulers allererster Vortrag zur Zahlentheorie in der Petersburger Akademie ist im Jahr 1732, das Ergebnis, das er präsentiert, zeigt, dass eine Vermutung Fermats falsch war, nämlich $2^{25}+1$ hat einen Faktor $641 .{ }^{18}$ Hiermit hat Euler innerhalb von drei Jahren die Goldbach aufgeworfene Frage, ob er Fermats Vermutung zu Zahlen der Form $2^{2^{n-1}}+1$ kenne, beantwortet. Euler kannte die Vermutung nicht, hat sich aber indessen in die Sache reingelesen, und die Vermutung mit einem Gegenbeispiel widerlegen können. Das

15 Dieses Problem wird öfters auch die Pell-Fermat-Gleichung genannt.

16 Fuss, Correspondance, S. 24.

17 Mehr Einzelkeiten dazu in H. Pieper (1993), „Euler's Contributions to the Four-Square Theorem“, Historia Mathematica, 20, S. 12-18.

18 L. Euler (1732/1738), „Observationes do theoremate quodam Fermatiano aliisque ad numeros primos spectantibus“, Comm. Ac. Petrop. 6, S. 103-107, auch in OO.I.2, S.1-5. 
Gegenbeispiel, der Faktor 641, hat Euler nach eigenen Angaben ,in diesen Tagen auf anderem Wege [als Probeteilungen] mit vielen Versuchen“ gefunden. Dieser andere Weg gibt Euler aber nicht an!

Die Mersenne- und Fermatzahlen haben einen Ruf in Sachen Rechenaufwand. 1603 hatte Cataldi die erste Primzahlliste überhaupt veröffentlicht (bis 750), nur um die Probeteilungen bei der Primzahlerkennung von Mersennezahlen zu erleichtern. Mit Hilfe dieser Liste konnte Cataldi zeigen, dass $2^{17}-1$ und $2^{19}-1$ Primzahlen sind, die Zahlen $2^{16}\left(2^{17}-1\right)$ und $2^{18}\left(2^{19}-1\right)$ mithin perfekte Zahlen.

Der Grund für Eulers Schweigsamkeit über seine Methode wird aber später verständlich. 1736, vier Jahre später, trägt Euler über Fermats kleinen Satz vor. ${ }^{19}$ Nach 6 Jahren ist es Euler endlich gelungen einen Beweis für $a+b$ teilt immer $a^{n}+b^{n}$ (mit $n$ ungerade) zu finden. Wie in einem noch späteren Aufsatz nachzulesen ist, kann man diesen Satz besonders effizient zur Primzahlerkennung gewisser Zahlen verwenden. ${ }^{20}$ Tatsächlich ist eine Fermatzahl $2^{2^{n-1}}+1$ der Form $a^{n}+b^{n}$ (mit $a$ gleich 2 und $b$ gleich 1), mithin kann man mögliche Teiler der Fermatzahl mit Fermats kleinem Satz der Form nach bestimmen und die Suche nach Teilern bedeutend einschränken. ${ }^{21}$

\section{Beobachtungen und Teilbeweise (1740-1756)}

Die Bewegungen in Eulers Beschäftigung mit den Eigenschaften der quadratischen Formen $\left(x^{2}+n y^{2}\right)$ kann man ebenfalls in den Briefen mit Goldbach nachspüren. Wahrscheinlich hat sich Euler zuerst mit den quadratischen Formen bekanntgemacht, als er 1729-1733 die Werke Fermats las. Eine eingehendere Studie scheint Euler aber erst in den frühen 40er Jahre aufgenommen zu haben, und ist in der Korrespondenz der frühen Berliner Jahre (ab 1741) wiederzufinden. Am 10. August 1741 fragt sich Goldbach im typischen Mischsprachen-Stil der Berliner Briefe:

Was halten Ew. Von dergl. Propositionibus: $(3 m+2) n^{2}+3$ kann niemals ein numerus quadratus seyn, positis pro $m$ und $n$ numeris integris quibuscunque? ${ }^{22}$

Euler fertigt Goldbach im erwidernden Brief mit einem 6-zeiligen Beweis dieser (einfachen) Behauptung ab, greift aber die Gelegenheit auf, schwerere Fragen ähnlicher Form zu beschreiben.

Ich habe vor langer Zeit auch solche ähnliche theoremata gefunden, als 4mn-m-1 kann nullo modo ein Quadrat seyn. Item 4mn-m-n [...] Von den divisoribus quantitatis $a a+m b b$, si $a$ und $b$ sint numeri inter se primi, habe ich auch curieuse proprietates entdeckt. ${ }^{23}$

Hier kommen die quadratische Formen in die Korrespondenz rein, als neue Entdeckung, aber ohne Beweise. In einem nächsten Brief, als Goldbach gesteht, diese von Euler behaupteten ,theoremata“ nicht beweisen zu können, zeigt Euler, wie die beiden von ihm gestellten Probleme zusammenhängen, er muss jedoch eingestehen, dass ein Beweis ihm

19 L. Euler (1736/1741), „,Theorematum quorundam ad numeros primos spectantium demonstratio“, Comm. Ac. Petrop. 8, S.141-146, auch in OO.I.2, S.33-37.

20 L. Euler (1747/1750), ,Theoremata circa divisores numerorum“, N. Comm. Ac. Petrop. 1, S. 20-48, auch in: OO.I.2, S. 62-85.

21 Eine Rekonstruktion von Eulers Methode durch Ed Sandifer findet man unter http://www.maa.org/news/howeulerdidit.html (Factoring F5, xxx).

22 Fuss, Correspondance, S. 104.

23 Ibid., S. 107. 
bis vor kurzem auch unerreichbar geblieben sei.

Dass $4 m n-m-1$ oder $4 m n-m-n$ niemals ein quadratum seyn können, konnte ich bis anjetzo auch nicht rigorose demonstriren, sondern ich hatte solches aus einem theoremate Fermatiano, worin behauptet wird, dass eine summa duorum quadratorum $a a+b b$ niemals per numerum formae $4 n-1$ divisibilis sey, hergeleitet. ${ }^{24}$ Von diesem letzteren Theorem hat Euler erst jetzt, zwischen den Briefen sozusagen, einen Beweis gefunden (mithilfe mit dem erst kürzlich bewiesenen kleinen Satz von Fermat), und mithin seine vorangehende, unbewiesene Behauptung ebenfalls bewiesen. Dieses Theorem ist aber jedoch nur einer unter vielen verwandten. Aus einem Brief einige Monate später nimmt man mehr als 20 ähnliche Theoreme, manche unbewiesen, die Euler über 6 Seiten seinem Freund Goldbach auftischt. ${ }^{25}$

Es folgt in der Korrespondenz ein komisches, wenn nicht peinliches Auf-der-StelleTreten in Sachen Zahlentheorie. Goldbach versucht selbst einen Beweis zu entwickeln, dass 4mn-m-1 oder 4mn-m-n niemals ein Quadrat sein können, scheitert jedoch an Eulers (richtigem) Skepsis. Im Dezember 1742, und nochmal vom März 1745 bis September 1745 schlägt Goldbach Beweiswege vor, die von Euler jedes Mal falsifiziert werden. Erst am 15. Oktober 1745 schreibt Euler - lesbar erleichtert - „nunmehr hat Ew. Demonstration [...] ihre völlige Richtigkeit".${ }^{26}$ Und Euler schreitet fort:

Ich habe noch einen grossen Vorrath von dergleichen theorematis, welcher Demonstration [...] gewiss nicht wenig zu Erweiterung dieser Wissenschaft beytragen würde. ${ }^{27}$

Es folgen 38 Sätze und ein ,etc.“ (Figur 3).

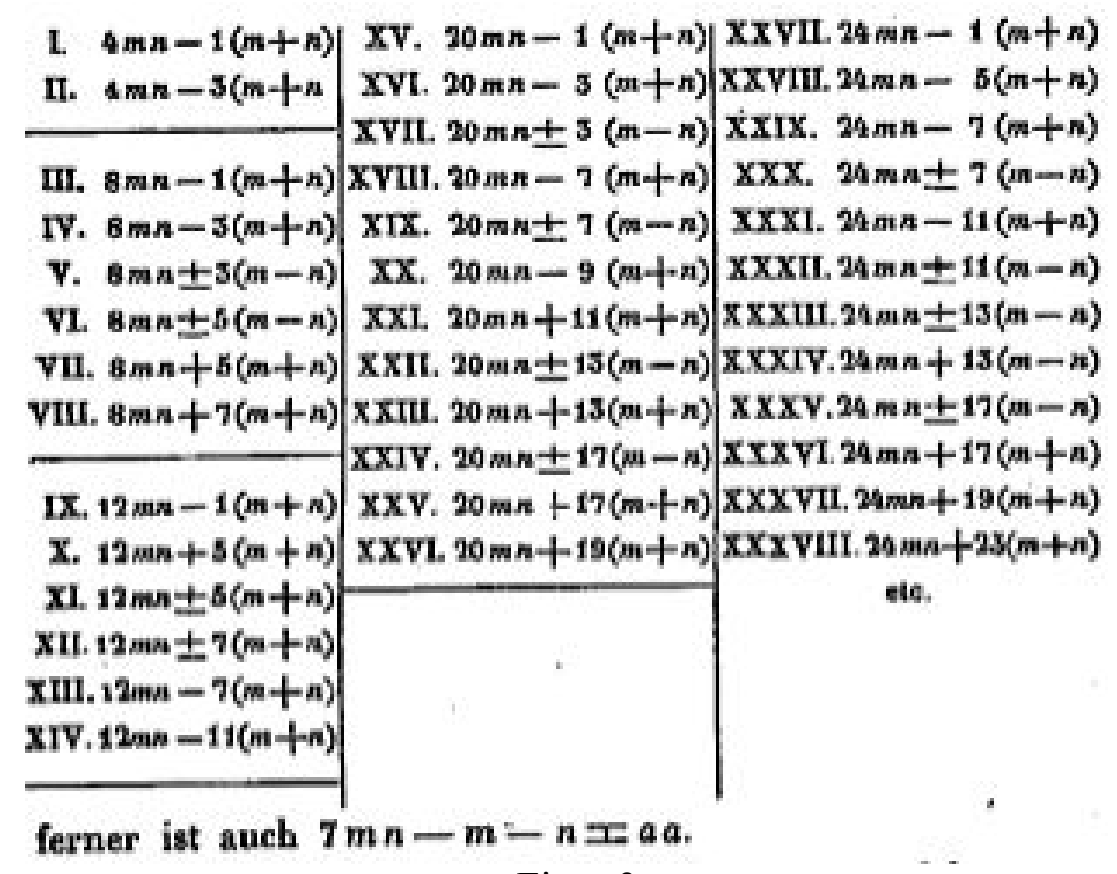

Figur 3

24 Ibid., S. 114.

25 Ibid., S. 145-150.

26 Ibid., S. 258.

27 Ibid., S. 259. 
Goldbach versucht zwar Eulers Sätzenflut zu bändigen und verallgemeinert die Frage: Für welche $e, f$, $g$ (alle ganze Zahlen) kann $e m n+f m+g n$ niemals ein Quadrat sein? ${ }^{28}$ Sein Versuch zur Lösung dieser allgemeinern Frage stößt jedoch wiederum auf Eulers Kritik, Goldbach habe ja vergessen $m$ und $n$ prim zu $f$ und $g$ (oder zu $p$ wenn $f=g=p$ ) zu nehmen. Dabei beschäftigen Euler im Moment vor allem die Formen emn-m-n (mit $e$ ein Parameter). Die Verseufzung folgt: „Es gibt in der Arithmetik eine grosse Menge solcher Sätze, an welchen Niemand zweifelt, ungeacht man dieselben nicht demonstriren kann. “29 Das Ergebnis dieser ersten Jahre von Eulers Forschung zu quadratischen Formen ist tatsächlich ein längerer Aufsatz, 1747 an der Akademie vorgetragen, zu welchem das Euler Archive berichtet: ,This seems to be a forgotten little article, hardly any proofs, but it contains a lot of conjectures that kept him going for the next several years. ${ }^{630}$ Der Aufsatz liest sich wie die Zusammenfassung der zweijährigen Korrespondenz zwischen Euler und Goldbach.

Sowohl Denkbewegungen in den Briefen, wie auch der Aufbau des 1747-Aufsatzes zeugen von Eulers Arbeitsmethode in der Zahlentheorie. In einem Aufsatz mit dem programmatischen Titel „Specimen de usu observationum in mathesi pura“ (1754/1761) hat Euler seine Ansichten und Methoden klar dargestellt:

Es wird nicht wenig paradox erscheinen, in jenem Teil der mathematischen Wissenschaften, den man gewöhnlich die reine Mathematik nennt, Beobachtungen große Bedeutung beizulegen, da der geläufigen Ansicht nach Beobachtungen auf physische Objekte beschränkt sind, welche die Sinne beeindrucken. Da wir die Zahlen auf den reinen Intellekt beziehen müssen, können wir kaum verstehen, wie Beobachtungen und Quasi-Experimente bei einer Untersuchung der Natur der Zahlen von Nutzen sein können. Doch sind tatsächlich [...] die heute bekannten Eigenschaften der Zahlen größtenteils durch Beobachtung entdeckt worden, und zwar lange bevor ihre Wahrheit durch strenge Beweise bestätigt wurde. [...] Die Art des Wissens, die nur von Beobachtungen gestützt wird und noch nicht bewiesen ist, muss sorgfältig von der Wahrheit unterschieden werden; sie wird, wie wir gewöhnlich sagen, durch Induktion gewonnen. [...] In der Tat sollten wir eine solche Entdeckung [per Beobachtung] als Gelegenheit dazu benützen, die entdeckten Eigenschaften genauer $\mathrm{zu}$ untersuchen und sie $\mathrm{zu}$ beweisen oder $\mathrm{zu}$ widerlegen; in beiden Fällen können wir etwas Nützliches lernen. ${ }^{31}$

Gerade der Fall $2 a a+b b^{32}$ liefert Euler das Material zur Veranschaulichung seines Arbeitsvorgangs. Eulers Arbeit beginnt mit Berechnungen und Beobachtungen, dann werden Vermutungen aufgestellt, die Euler zu beweisen sucht, und wenn mehrere Vermutungen oder bewiesene Sätze sich ähneln (wie in fig. 3), so kann man sich langsam der Generalisierung nähern.

28 Ibid. S. 276.

29 Ibid. S. 302

30 L. Euler (1748/1751), ,Theoremata circa divisores numerorum in hac forma paa \pm qbb contentorum“, Commentarii academiae scientiarum Petropolitanae 14, S. 151-181, auch in OO.I.2 S. 194-222.

31 L. Euler (1756/1761), „Specimen de usu observationum in mathesi pura“, N. Comm. Ac. Petrop. 6, S.185-230, auch in OO.I.2, S. 459-492. Hier S. 185-6, übersetzt von Wulf-Dieter Geyer. Eulers Wortbildung „Quasi-Experiment“ aus der Einleitung zu diesem Aufsatz wurde vom Mathematiker und Philosophen Imre Lakatos in dem Buch Proofs and Refutations (1976) aufgegriffen um die „empirische“ Ansicht in der Mathematik zu fassen, nämlich, den Quasi-Empirismus in der Mathematik.

32 Ein Kommentar und Teilübersetzung von Ed Sandifer, der die mathematischen Ergebnisse des Aufsatzes erörtert, kann man finden unter http://www.maa.org/news/howeulerdidit.html $(2 a a+b b$, January 2006). 
Am Ende von Eulers erster intensiven Periode der zahlentheoretischen Forschung steht ein unvollendeter Aufsatz ${ }^{33}$, den André Weil öfters als einen 50 Jahre früher geschriebenen, aber leider gescheiterten Versuch, ein Lehrbuch zur Zahlentheorie zu schreiben, gewertet hat. Datiert wird diese Skizze auf das Jahr $1756 .{ }^{34}$ Dieser Text, die Doctrina numerorum, ähnelt den ersten drei Sektionen von den Disquisitiones Arithmeticae, die C.F. Gauss 1801 veröffentlichen wird, aber bei der Niederschrift scheiterte Euler an Sätzen, die er nicht beweisen konnte. Dieser Aufsatz ist nahezu typisch für Eulers zahlentheoretische Arbeiten in der zweiten Hälfte seiner Berliner Periode (1756-1766), sie versuchen die gefundenen Resultate $\mathrm{zu}$ bündeln, und einen Überblick zu gewinnen. Die wirklichen Fortschritte in der Theorie scheint Euler zu dieser Zeit nicht mehr zu gewinnen. Erst als ein neuer Dialog entsteht, ab 1771 mit Lagrange und anderen, schwingt sich Euler wieder zu neuen Arbeiten, Funden und Niederschriften auf.

\section{Der verzögerte Dialog mit Lambert und der Berliner Akademie (1771-1775)}

Bekanntlich wurde Euler, nach einer misslungenen Operation an seinem Auge, 1771 fast vollständig blind. Doch ist die Periode 1771-1775 eine besonders fruchtbare, besonders auf dem Gebiet der Zahlentheorie. Das ist daraus zu erklären, dass, einerseits, Euler, zum ersten Mal seit der Korrespondenz mit Goldbach, jetzt im Dialog mit Kollegen (Lagrange, Lambert, ...) sich auf zahlentheoretische Fragen fokussieren konnte, andererseits, dass Euler sich schon schnell eine effiziente Arbeitsweise zueignete, die mit seiner Fast-Blindheit klarkam.

Wie Euler in seinen letzten Lebensjahren sein wissenschaftliches Arbeiten organisierte, hat P.H. Fuss, Sohn des letzten Assistenten Eulers, eindringlich und detailgetreu beschrieben:

Euler avait dans son cabinet une grande table qui occupait tout le milieu de la pièce et dont le dessus était recouvert d'ardoise. C'est sur cette table qu'il écrivait, ou plutôt indiquait ses calculs en gros caractères avec de la craie. Chaque matin, son élève se présentait chez lui pour lui faire lecture soit de sa vaste correspondance [...] soit de quelque nouvel ouvrage digne d'attention; on s'entretenait [...]. Quand la table était couverte de calculs, ce qui arrivait souvent, le maître confiait au disciple ses conceptions toutes fraîches et récentes et lui exposait la marche de ses idées et le plan général de la rédaction, en lui abandonnant le soin du développement des calculs, du choix d'exemples et de l'exécution des détails; et ordinairement, celui-ci lui apportait dès le lendemain le croquis du mémoire inscrit dans le grand livre [Adversaria mathematica]. Ce croquis approuvé, la pièce était rédigée au net et presentée immédiatement à l'Académie. ${ }^{35}$

Anhand der ihm vorgelesenen Essays, Bücher und Briefe kann man tatsächlich Eulers Arbeit chronologisch nachvollziehen.

33 L. Euler (1756/1862), „Tractatus de numerorum doctrina Capita XVI, quae supersunt“, in: Opera Posthuma (Leipzig, Berlin: Teubner), Band 1, S. 3-75, auch in OO.I.5 S. 182 - 283.

34 Weil, „Oeuvre arithmétique“, S. 112.

35 Fuss, Correspondance, S. XLIV-XLV. 
1766 hatte Euler Berlin verlassen und war nach Sankt-Petersburg zurückgekehrt. In Eulers Verhältnissen und Missstimmungen mit seinen Mathematiker-Kollegen sind die Gründe seines Umzugs zu finden. ${ }^{36}$ Zum einen war Euler verstimmt, dass Friedrich der Grosse ihm den Vorsitz der Berliner Akademie nach dem Tod Maupertius' nicht gewähren wollte, sondern, schlimmr noch, den Vorsitz seinem Erzrivalen in Sachen Mathematik, Jean Le Rond d'Alembert, anbot. Obwohl dieser das Angebot ausschlug, ernannte Friedrich der Grosse immer noch nicht Euler zum Vorsitzenden, sondern nur zum Vorsitzenden ad interim. Zum anderen hatte Eulers Beziehung zu Koller, der als Trésorier für die wichtigste Einnahmequelle der Akademie, das Privileg des Almanachs, zuständig war, viele Akademiemitglieder verstört. Insbesondere Johann Heinrich Lambert, 1765 nach Berlin berufen, wehrte sich in der Prüfungskommission gegen Eulers Absprachen mit Koller. Friedrich der Grosse gab Lambert recht mit den berühmten Worten: „Quoique je n'aye pas appris à calculer les courbes, je sais pourtant, mon cher Euler, que pour mon académie, dix-sept reisdallers valent mieux que treize. ${ }^{\text {“37 }}$ Unter diesen Umständen darf es verwundern, dass Euler gerade in den Sankt-Petersburger Jahren, zweite Periode, einen intensiven Dialog mit den Berliner MathematikernAkademikern beginnt. Allerdings ist J.H. Lambert von diesem Dialog ausgeschlossen ... Selbstverständlich blieb Euler mit Louis Joseph Lagrange im Dialog. 1754 hatte Lagrange den Briefwechsel mit Euler angefangen, und als Euler Berlin hinter sich ließ und somit die Akademie seines Direktors der mathematischen Klasse beraubte, so schlug er Lagrange als seinen Nachfolger vor. 1766 wurde Lagrange dann tatsächlich nach Berlin berufen. Der Briefwechsel wurde diesem guten persönlichen Verhältnis entsprechend in den Jahren 1766 bis 1775 weitergesetzt. Als Lagrange dann 1767 (1769 veröffentlicht) seine vollständige Lösung der Pell-Fermat-Gleichung schreibt ${ }^{38}$, eine große Arbeit mit der er auf Eulers Ergebnisse weiterbaut, reagiert Euler:

Comme je ne suis pas en état de les [Lagranges Essais] lire moi-même, j'ai prié notre habile M. Lexell de m'en faire la lecture, que j'ai entendue avec la plus grande avidité. $^{39}$

Euler hat auch Lagranges Beispiele der neuen Methode durchgerechnet, obwohl ein gewisses Beispiel ihm „quelque embarras"' bereitet hat. Lagrange (in einem leider nicht bewahrten Brief) antwortet ausführlich und schreibt das Beispiel mit allen rechnerischen Details hin. Leider reicht auch diese Didaktik für den fast blinden Euler nicht mehr aus:

Je me suis fait lire toutes les opérations que vous avez faites sur la formule et je suis entièrement convaincu de leur solidité; mais étant hors d'état de lire ou d'écrire moi-même, je dois vous avouer que mon imagination n'a pas été capable de saisir le fondement de toutes les déductions que vous avez été obligé de faire et encore moins de fixer dans mon esprit la signification de toutes les lettres que vous y avez introduites. Il est bien vrai que de semblables recherches ont fait autrefois mes délices et m'ont coûté bien du temps; mais à présent je ne saurais plus entreprendre

36 Siehe dazu, K.-R. Biermann (1985), „Wurde Leonhard Euler durch J.H. Lambert aus Berlin. Vertrieben?“, in: Festakt und Wissenschaftliche Konferenz 1983 in Berlin. Berlin: Akademie-Verlag, S. 91-99.

37 Dieudonné Thiébault (1804), Mes souvenirs de vingt ans de séjour à Berlin; ou Frédéric le Grand, sa famille, sa cour, son gouvernement, son académie, ses écoles, et ses amis littérateurs et philosophes. Paris: Buisson, Band 5, S.10.

38 Lagrange, J. de (1767/1769), „Sur la solution des Problèmes indéterminés du second degré“, Histoire de l'Académie Royale des Sciences et des Belles-Lettres de Berlin 2, S. 165-310.

39 Lagrange, J. De (1867-1892), Oeuvres., hg. von M.J.-A Serret. Paris: Gauthier-Villars, 14 Bände.Briefwechsel Euler-Lagrange im 14. Band, hier S. 214. 
que celles que je suis capable de développer dans ma tête et souvent je suis obligé de reccurir à un ami pour exécuter les calculs que mon imagination projette. ${ }^{40}$

Eulers Beschreibung, warum er Lagranges Beweis und Berechnung nicht nachvollziehen kann, ist bemerkenswert: „de fixer dans mon esprit la signification de toutes les lettres que vous y avez introduites". Auch ein nur oberflächlicher Leser von Lagranges Arbeiten kennt dieses Problem. Lagrange ist ein Meister des Buchstabenkalküls, und es fällt vielen schwer, alle auftretenden Lettern und Indices im Kopf zu behalten, geschweige denn, wenn man sie nur über das Gehör vernehmen kann. Vielleicht deswegen dialogiert Euler in dem Zeitraum 1770-1773 nicht sosehr mir Lagranges großer Arbeit sondern eher mit Arbeiten der anderen Berliner Akademiemitglieder.

1770 hatte Johann Heinrich Lambert ein Buch, Zusätze zu den logarithmischen und trigonometrischen Tafeln, veröffentlicht, dessen Einführuing als Programmschrift gelten kann. In dieser Einführung rief Lambert Mathematiker und Liebhaber der Mathematik auf, zusammen an einer Erweiterung und Befestigung der Kenntnisse der ganzen Zahlen $\mathrm{zu}$ arbeiten. Zum einen wollte Lambert mit seinem Aufruf, die Faktoren- und Primzahltabelle bis auf die Million erweitern, zum anderen wollte er eine Theorie der Zahlen fördern, denn die gab es anno 1770 noch nicht.

Ich habe mich zu diesem Ende [der Primzahlerkennung] so wie auch zu andern Absichten um die Theorie der Primzahlen näher umgesehen, und da fand ich freylich nur einzelne abgebrochne Stücke, ohne sonderlich Anschein, daß dieselbe so bald sollten zusammengehängt und zum förmlichen System gemacht werden können. Euclid hat wenig, Fermat einzelne meistens unbewiesene Sätze, Euler einzelne Fragmente, die ohnehin von den ersten Anfängen weiter entfernet sind und zwischen sich und den Anfängen Lücken lassen. ${ }^{41}$

Lambert war nicht der erste, der eine Theorie der Zahlen begründen wollte. Fermat hatte schon dazu aufgerufen, und vor allem der englische Mathematiker John Wallis (16161703) hatte öfters diesen Wunsch ausgesprochen. In Wallis' Tradition - und nicht in der Tradition Diophants und Fermats - steht Lambert. ${ }^{42}$ Im Gegensatz zu der von den alten Griechen herrührenden Tradition (perfekte Zahlen, Diophant), konzentriert sich die Wallis-Lambert-Tradition eher auf die Aufschreibesysteme der Zahlen, auf die Anatomie der Zahlen, wie es im 18. Jh. manchmal genannt wurde. ${ }^{43}$ Statt Diophantischer Probleme oder perfekter Zahlen, Probleme die Diophant-Fermat-Tradition eine zentrale Stelle innehaben, konzentriert sich die Wallis-Lambert-Tradition eher auf Fragen über die Zahlensysteme, und auf abstraktere Probleme der Zahlenkenntnis, wie die Faktorisierung. Ein für Wallis und Lambert interessantes Problem waren die Regelmäßigkeiten der Ziffern in verschiedenen Zahlsystemen. So ist z.B. 1/7 als Dezimalbruch 0,142857142857..., und 2/7 0,285714285714... Beide Dezimalbrüche sind periodisch, und beide Brüche haben sogar dieselbe ,sich wiederholende Ziffernfolge, sei es um 2 Ziffern versetzt.

40 Lagrange, Oeuvres, Band 14, S. 219.

41 J.H. Lambert (1770), Zusätze zu den logarithmischen und trigonometrischen Tabellen. Berlin: Spener'sche Buchhandlung, S. 20.

42 G.W. Leibniz steht mit seinen vereinzelten Studien zu zahlentheoretischen Fragen ebenfalls in Wallis' Tradition. Wallis selbst scheint vor allem von John Pell (1611-1685) beeinflusst zu sein, aber leider verbietet die Kargheit letzterer beim Publizieren weitere Bestätigung.

43 Mehr Einzelheiten über diese Geschichte und die involvierte Mathematik findet man in: M. Bullynck (2008), „From Anatomia Numerorum to Higher Arithmetics A History of Factor Tables with Notes on the Birth of Number Theory 1668-1817“, erscheint demnächst in Revue d'Histoire des Mathématiques. 
Lambert hatte sich mit diesem Problem schon zweimal auseinandergesetzt, und sein Kollege an der Akademie, Johann III Bernoulli, veröffentlichte 1771 auch einen Aufsatz zum Thema der repetitiven Dezimalbrüchen und deren Anwendung bei Faktorisierung gewisser großer Zahlen (der Form $\left.10^{n}+1\right) \cdot{ }^{44}$ Ebenfalls von Lamberts Programm für die Zahlenthoerie angespornt, schreibt 1773 ein anderers Akademiemitglied, Nikolaus von Béguelin, einen Aufsatz über eine neue Methode, zu testen, ob Mersennezahlen Primzahlen seien oder nicht. ${ }^{45}$ Béguelins Ansatz ist ebenso einfach wie vernünftig: Er schreibt die Mersennezahlen nicht mit dezimalen Ziffern, auch nicht mit binären Ziffern, sondern mit den binären Exponenten. ${ }^{46}$ Zum Beispiel: Die Mersennezahlen $2^{n}-1$ und die Zahl $2^{n}+1$ mit $n=4$, schreibt man dezimal als 15 und 17, binär als 1111 und 10001, und in Béguelins Zahlensystem als 0.1.2.3. und 0.5. Das heißt, die Stellen im Binärzahl, wo eine 1 steht, diese schreibt man hin. Nach Béguelin verbindet dieses Zahlensystem verschiedene Vorteile: Es hat die Bündigkeit des Binärsystems, und die Permutierfähigkeit der algebraischen Buchstaben (d.h. ab ist gleich ba; oder bei Béguelin, 0.5. ist gleich 5.0.). ${ }^{47}$ Eben solche Art von Überlegungen zeichnet die WallisLeibniz-Tradition aus. ${ }^{48}$ Indem er diese Eigenschaften ausnutzt, findet Béguelin ein neues Verfahren (unter Zuhilfenahme einiger Ergebnisse Eulers) für das Primtesten von Mersennezahlen.

Eben auf diese zwei Aufsätze reagiert Euler, vielleicht weil er die figurative Seite der Probleme leichter im Kopf visualisieren kann, als Lagranges verwickelte Lettern und Indices. 1772 lässt Euler Johann III Bernoulli schreiben, er habe mit Freuden dessen Aufsatz gelesen. ${ }^{49}$ Und Euler fügt hinzu, dass er ein noch einfacheres Kriterium zur Zerlegung der Zahlen der Form $10^{n}+1$ als dasjenige Bernoullis geben kann. Dieses Kriterium aber, beruht auf ein Prinzip, ,dessen Beweis noch nicht bekannt ist. “50 Es handelt sich um das Gesetz der quadratischen Reziprozität, das erst A.-M. Legendre 1798 formulieren, und C.F. Gauss 1801 gleich zweimal beweisen wird. ${ }^{51}$ Euler selbst formuliert das Gesetz niemals, obwohl er verschiedene „kleinere“ Fälle des Gesetzes formuliert, und einige auch beweist. So zeigt Euler im oben erwähnten Aufsatz „De usu observationum...", dass das Gesetz für -1 gültig ist. Für die Entwicklung der

44 Johann III Bernoulli (1771/1773), „Sur les fractions décimales périodiques. Suivi de: Recherches sur les diviseurs de quelques nombres très grands“, Nouveaux Mémoires de l'Académie Royale des Sciences et Belles-Lettres de Berlin, S. 273-337.

45 Nikolaus von Béguelin (1773/1775), „Solution particulière du Problème sur les nombres premiers“, Nouveaux Mémoires de l'Académie Royale des Sciences et Belles-Lettres de Berlin, S. 300-322.

46 Nikolaus von Béguelin (1770/1772), „Essais sur un Algorithme déduit du principe de la raison suffisante", Nouveaux Mémoires de l'Académie Royale des Sciences et Belles-Lettres de Berlin, S. 296352.

47 Béguelin, Algorithme, S. 297.

48 Obwohl man bemerken sollte, dass diese Überlegungen auch in Leibniz-Wolff'scher Tradition einer cognitio symbolica stehen

49 L. Euler (1772/1774), „Extrait d'une lettre de M. Euler le père à M. Bernoulli“, Nouveaux Mémoires de l'Académie Royale des Sciences et Belles-Lettres de Berlin, S. 35-36.

50 Ibid., S. 36.

51 Das Gesetz lautet folgendermaßen: Wenn eine Zahl $a$ Rest eines Quadrats nach Teilung durch $b$ ist, dann ist $b$ auch Rest eines Quadrats nach Teilung durch $a$, ausgenommen den Fall, dass sowohl $a$ als $b$ der Form $4 n+3$ sind (dann ist $a$ quadratischer Rest von $b$, und $b$ nicht-quadratischer Rest von $a$ und umgekehrt). So ist, z.B., 12 Rest von $81=9^{2}$ nach Teilung durch 23 , und ist 23 Rest von $143=13^{2}$ nach Teilung durch 12 (oder auch, wenn man eine 12 mehr aus 23 holt, ist 11 Rest von 143 nach Teilung durch 12). 
Zahlentheorie im 19. Jh. wird das Reziprozitätsgesetz und dessen Erweiterungen eines der wichtigsten Themen sein, Katalysator auch von vielen Studien. Die Lektüre von Bernoullis Text verwertet Euler für sich auch in einigen Vorträgen an der Petersburger Akademie, und später mit dementsprechenden Veröffentlichungen. ${ }^{52}$

Auf Béguelins Untersuchungen reagiert Euler ebenfalls ${ }^{53}$, und erneut greift er in seiner Antwort auf ältere, zum Teil „empirische“ Untersuchungen zurück, versucht diese zu verallgemeinern, und formuliert so Vermutungen, die für die spätere Entwicklung der Zahlentheorie wichtig werden sollen. Mai 1778 schreibt Euler, dass Béguelin in seiner Arbeit sehr wohl die Eigenschaft benutzt, dass eine Primzahl nur in genau einer Weise als Summe zweier Quadrate geschrieben werden kann $\left(A=x^{2}+y^{2}\right)$, aber dass diese Eigenschaft auch anderen Formen $x^{2}+n y^{2}$ (mit $n>1$ ) eigen ist. Dann listet Euler 65 Zahlen $n$ (die größte ist 1848), für welche die Form $x^{2}+n y^{2}$ ( $x$ und $y$ prim zueinander) diese Eigenschaft hat, und nennt diese Zahlen ,nombres convenables“, geschickte Zahlen (d.h. geschickt zum Faktorisieren). Außer diese 65 hat Euler keine gefunden, aber einen Beweis dazu hat er nicht. Béguelin zeigt seinem Kollegen Lagrange diesen Brief Eulers, und Lagrange ist besonders an diesen ,geschickten Zahlen“ interessiert, und fragt Béguelin, ob er denn Euler nicht um mehr Kommentar bitten kann. Über seinen Assistenten, Nikolas Fuss, richtet Euler die Antwort aus. ${ }^{54}$ Bis heute ist die Frage ungeklärt, ob es neben den von Euler (und später von Gauss) angegebenen „,nombres convenables" noch andere gibt. Der kleine Briefwechsel mit Béguelin gibt, ähnlich wie bei Bernoulli, ebenfalls Anlass zu mehreren Aufsätzen, in denen Euler große Zahlen mithilfe der geschickten quadratischen Formen faktorisiert, Aufsätze die größtenteils erst nach Eulers Tod erscheinen. ${ }^{55}$

\section{Eulers Algebra (dt. 1770 und fr. 1775) und die Promotio scientiae numerorum (1775)}

Eulers Vollständige Anleitung zur Algebra,1770 deutsch ${ }^{56}$ und russisch erschienen und 1774 in französischer Übersetzung von der Hand Johann III Bernoullis mit Zusätzen von Lagrange $^{57}$, darf in gewisser Hinsicht als die Summa, als die überblickende Synthese eines Lebens in der Mathematik, besonders in der Arithmetik, gelten. Und die französische Übersetzung führt gerade da, wo Euler aufhört, weiter, und fügt die Untersuchungen derer, die auf Eulers Arbeiten weitergebaut haben (Lambert, Lagrange), an Eulers Elémens d'Algèbre zu - eine „Modernisierung“ und Aktualisierung.

Wenn Euler Ende der 60er Jahre seine Anleitung zur Algebra diktiert, so greift er gleich auf seine Kindheitserinnerungen zurück und benutzte Stifels Coss-Edition als Hauptquelle für die Fragen und Probleme, die den Text illustrieren sollten. ${ }^{58}$ In diesem

52 Dies sind die Arbeiten E449, E552, E554, E557, alle aus dem Jahr 1772.

53 L. Euler (1778/1779), „Extrait d'une lettre de M. Euler le père à M. Beguelin en mai 1778“, Nouveaux M emoires de l'Académie Royale des Sciences et BellesLettres de Berlin, S. 337-339.

54 N. Fuss (1778/1779), „Extrait d'une lettre de M. Fuss à M. Beguelin écrite de P' etersbourg le 19-20 juin 1778“, Nouveaux Mémoires de l'Académie Royale des Sciences et Belles-Lettres de Berlin, S. 340346.

55 Dies sind die Arbeiten E699, E708, E715, E718, E719, E725, alle fertiggeschrieben, aber im Nachlass der letzten Lebensjahre gefunden und dann veröffentlicht.

56 L. Euler (1770), Vollständige Anleitung zur Algebra. St.-Petersburg: Kays. Akad. d. Wiss., 2 Bände.

57 L. Euler (1774), Elémens d'Algèbre. Lyon: Bruyset, 2 tômes.

58 Dies hat Albrecht Heeffer („The origin of problems in Euler's Algebra“, Bulletin of the Belgian 
Sinne wiederholt er mit seinem Assistenten, dem die Algebra beim Anfang der Niederschrift unbekannt war, was sein Vater mit ihm gemacht hat. Die zeitgenössischen Rezensenten ist dieser Aspekt des diktierenden blinden Eulers nicht entgangen, im Gegenteil, sie verstanden es als die beste Werbung für Eulers Buch:

ein Lehrbuch von der Algebra aus dem man ohne andere Beyhülfe leicht und gründlich die Algebra erlernen könnte. Er machte von der Ausführung dieses Gedankens selbst die Probe, mit dem, dem er das Buch in die Feder dictirte, einem jungen Menschen [...]. Dieser lernte in kurzer Zeit, schwere Buchstabenrechnungen führen, und algebraische Aufgaben fertig aufzulösen. ${ }^{59}$

Mithin kann sich jeder fleißige Leser Eulers in den Zustand des Schreibers versetzen, und sich selbst die Algebra beibringen. Eulers Algebra behandelt im ersten Band die Größen, die vier Rechnungsarten, Bruchzahlen, Logarithmen und Proportionen; im zweiten Band Gleichungen in $x$ ersten, zweiten, dritten und vierten Grades und deren Auflösungen, und schließlich unbestimmte Gleichungen in $x$ und $y$ erster und zweiter Ordnung (also: Diophantische Gleichungen erster und zweiter Ordnung).

Wie traurig Eulers Blindheit auch, indirekt hat man ihr nicht nur die Algebra zu danken, sondern auch Lagranges Zusätze zur bedeutend umgearbeiteten französischen Übersetzung der Algebra. Schon schnell nach dem Erscheinen von Eulers Algebra hatte Jean le Rond d'Alembert seinem Freund J.-L. Langrange gegenüber den Wunsch geäußert, man möge dieses gute Lehrbuch ins französische übersetzen. Tatsächlich übernahm die Berliner Akademie die Aufgabe, jenes Werk zu übersetzen; Johann II Bernoulli zeichnete für die Übersetzung, Umordnung und Fussnoten, Lagrange für seine famösen Addtions.

In diesen polierte und ziselisierte Lagrange seine Theorie der quadratischen Formen. Vielleicht zum Teil, weil Langrange sich erhoffte, Euler den Zugang zu seiner Theorie zu erleichtern, denn in einem Brief an den Marquis de Condorcet schreibt Lagrange:

Quant à mes recherches sur les problèmes indéterminés, je vous suis d'autant plus obligé d'avoir pris la peine de les lire, que je vous crois le seul qui m'ait fait cet honneur; car M. Euler [...] m'a mandé, que la perte de sa vue ne lui ayant pas permis de lire mes Mémoires, il n'avait cependant pas manqué de se les faire lire, mais qu'il lui avait été impossible de suivre mes raisonnements et mes calculs. J'ai tâché de les rendre un peu plus clairs et plus concis dans des additions que j'ai faites à la traduction française de l'Algèbre allemande d'Euler. ${ }^{60}$

Tatsächlich bilden Lagranges Additions eine weitere Ziselierung von Lagranges Synthese aus 1767 zur Pell-Fermat-Gleichung.

Im Wesentlichen aber fand Lagrange Eulers Algebra uninteressant (denn als Lehrbuch enthält es vor allem bekannte Sachen), den Teil über unbestimmte Analysis ausgenommen. ${ }^{61}$ Dennoch sah Lagrange eine Chance, eine Übersetzung von Eulers Algebra dazu zu nutzen, dass eigentlich zugleich auch ein Lehrbuch zur unbestimmten Analyse, zu Diophantischen Problemen erscheinen könnte. In dem Avertissement zu den Additions, welche Langrange an Eulers Buch hinzufügt, schreibt Lagrange:

On n'avait aucun [livre] où cette science [1'Analyse de Diophante] fût traitée d'une

Mathematical Society - Simon Stevin, Band13 Nr. 5 (2007), S. 949-952) mittels extensiven Quellenstudiums für Eulers Probleme nachgewiesen.

59 A.G. Kästner, „Rezension zu Eulers Algebra“, Göttingische Anzeigen von gelehrten Sachen, 14. Stück 1771, S. 113.

60 Lagrange, Oeuvres, Band 14, S. 4.

61 So in den Briefen an d'Alembert: Lagrange, Oeuvres, Band 13, S. 181 und 191. 
maniere méthodique, \& qui renfermât \& expliqât clairement les principales regles connues jusqu'ici pour la solution des problêmes indéterminés. Le Traité précédent [l'Algèbre d'Euler] réunit ce double avantage; mais pour le rendre encore plus complet, j'ai cru devoir y faire plusieurs additions ${ }^{62}$

Lagranges Extraseiten sind umfassend, fast 300 Seiten, und deswegen nimmt die französische Übersetzung den deutschen 1. Band mit der 1. Hälfte des 2. Bandes (bestimmte Gleichungen in $x$ ) für Tôme 1 zusammen. Tôme 2 ist dann ausschließlich der unbestimmten Analyse gewidmet: Seite 1 bis 370 von Eulers Hand, Seite 371 bis 667 von Lagranges Hand. So wie Euler die Teile seiner Algebra nach der Art der benutzten Zahlen geordnet hatte (ganze Zahlen, Bruchzahlen, Wurzeln), so fügt Langrange noch ein extra Kapitel und eine extra „Zahlensorte“ hinzu: Kettenbrüche, Lagranges geliebte Technik zur Lösung unbestimmter Fragen.

Johann III Bernoulli hingegen beschränkt sich als Übersetzer auf wenige Fussnoten, die nach Bernoullis Aussage - ,peuvent d'aillieurs répandre du jour sur différens points d'histoire des Mathématiques, \& faire connoitre un grand nombre de tables subsidiaires peu connues." (S. xvj) In diesen Fussnoten berichtet Bernoulli aber von Lamberts Faktorentabellen, von Bernoullis eigenem Versuch, periodischen Dezimalbrüchen einen theoretischen Rahmen zu geben, ... Kurz, er schreibt in den Fussnoten das Update zu Eulers Buch. In diesem Sinne ist Eulers Algebra mitsamt der Übersetzung als eine Summa von Eulers Arbeit zu verstehen, von den Anfängen bei Stifel bis zu der nach Euler kommenden Generation.

Wenn es aber um Eulers Streben, an den Grenzen des Bekannten in Sachen Diophantischer Mathematik zu arbeiten, ist ein anderer Text zwar nicht die Summa sondern das nec plus ultra, das Euler in der Zahlentheorie noch vor seinem Tode in 1783 schafft. Während Lagrange die Additions schreibt, erzielt dieser noch weitere Fortschritte und Simplifizierung, die er in zwei Teilen 1773 und 1775 in den Mémoires der Berliner Akademie veröffentlicht, unter dem einfachen aber bedeutenden Titel „Recherches Arithmétiques“.63 Mit den „Recherches“ kommt eine Periode in der Forschung quadratischer Formen zu Ende. Erst mit den „Recherches“ wird Legendres Essai d'une théorie des nombres (1798) und Gauss' Disquisitiones Arithmeticae (1801) möglich, die beiden Werke, die die moderne Zahlentheorie begründen.

Als Euler den ersten Teil von Lagranges Aufsatz zu Gehör bekommt, sieht er sofort die Wichtigkeit von Lagranges neuesten Untersuchungen ein, und diktiert seinem Assistenten einen Aufsatz, der 1775 der Akademie vorgelegt wird. ${ }^{64}$ Der Aufsatz fängt mit einer Lobrede auf Lagranges 1773-Text an, mit dem Lagrange „Licht in die Wissenschaft der Zahlen, die bisher in Schatten gewickelt war, gebracht hat." ${ }^{65}$ Vieles aber bei Lagrange könnte - laut Euler - noch klarer ausgedrückt werden, wenn man Sätze aufstellt, die zwar allgemeiner sind, aber deren Wahrheit Euler nur per Induktion begründen kann. Mitten in Eulers Text steht dann auch ein theorema demonstrandum ${ }^{66}$ ein zu beweisender Satz, der gewisse Punkte bei Lagrange bedeutend vereinfachen kann, dessen Beweis aber noch aussteht. Der Titel von Eulers Aufsatz ist ebenso explizite wie derjenige Lagranges: „ De insigni promotione scientiae numerorum", über eine

62 Euler, Elémens, tôme 2, S. 374 (zitiert nach der 2. Ausgabe von 1792).

63 Lagrange, „Recherches Arithmétiques“, Nouveaux Mémoires de l'Académie Royale des Sciences et Belles-Lettres, 1773, S. 265-312 und 1775, S. 323-356.

64 L. Euler (1775/1785), „De insigni promotione scientiae numerorum“, Opuscula Analytica. Petersburg: Kais. Ak. d. Wiss., Band 2, S. 275-314, auch in OO.I.4 S. 163-196.

65 Euler, Promotione, S. 275.

66 Ibid. S. 308. 
bedeutende Beförderung der Wissenschaft der Zahlen.

Genau diese beiden Arbeiten, Lagrange „Recherches“ und Eulers „Promotione“ stehen am Wendepunkt, wo die Periode von Bachet und Fermat bis Euler und Lagrange abgeschlossen wird und eine neue Periode, die Legendres und Gauss', anfängt. Während Lagranges Text Gauss' wichtigen Begriff der Klasse vorbereitet, regt Eulers Artikel Untersuchungen bei Legendre und Gauss an, die Gauss zum Genus-Konzept führen wird. Mit der Bestimmung dieser beiden Begriffe kann sich Gauss an die Niederschrift der Sektion V der Disquisitiones Arithmeticae setzen, und neue Generationen von Mathematikern inspirieren. In einem durchschossenen Buch, auf dessen leeren zwischengefügten Seiten Gauss in seinen frühen Jahren manche Notiz zu den Disquisitiones schrieb, ist auf der ersten Seite einen Spruch Lagranges zu finden, am Ende jedoch ein längeres Zitat Eulers, das hier als allgemeines Fazit zu Eulers Arbeit in der Zahlentheorie stehen darf:

Wenn wir auf ihre Nützlichkeit blicken, scheinen mathematische Uberlegungen auf zwei Klassen zurückgeführt werden zu müssen; in die erste sind diejenigen aufzunehmen, die sowohl für das tägliche Leben wie für die anderen Künste irgendeinen bedeutenden Vorteil bringen [...] Die zweite Klasse aber umfasst diejenigen Überlegungen, die, auch wenn sie mit keinem bedeutenden Vorteil verbunden sind, dennoch so beschaffen sind, dass sie Gelegenheit bieten, die Grenzen der Analysis hinauszuschieben und die Kräfte unseres Geistes zu schärfen. ${ }^{67}$

67 Zitiert aus: K. Reich (2005), „Gauß' geistige Väter: nicht nur 'summus Newton', sondern auch 'summus Euler',,, in: ,, Wie der Blitz einschlägt, hat sich das Räthsel gelöst“ Carl Friedrich Gauß in Göttingen, hg. von E. Mittler (Göttingen: Niedersächsische Staats- und Universitätsbibliothek Göttingen), S. 105115. Hier S. 107-108. 